\title{
Non-alcoholic fatty liver disease frequency and associated factors at admission of acute stroke
}

\author{
Takahisa Mori $^{1} \odot \cdot$ Kazuhiro Yoshioka ${ }^{1} \cdot$ Yuhei Tanno $^{1}$
}

Received: 23 June 2021 / Accepted: 1 September 2021 / Published online: 15 September 2021

(c) The Author(s) 2021

\begin{abstract}
Background/purpose of the study If non-alcoholic fatty liver disease (NAFLD) frequency is very high in stroke patients, NAFLD may be a risk factor for stroke and identifying factors of NAFLD presence may lead to stroke prevention. This retrospective study aimed to investigate whether NAFLD frequency was very high and identify factors associated with NAFLD presence at acute stroke admission.

Methods We included stroke patients aged 40 - 79 years who (1) were admitted from 2016 to 2019, within $24 \mathrm{~h}$ of onset; (2) underwent abdominal ultrasonography; and (3) underwent blood examination of biomarkers. We evaluated the frequency and significant factors of NAFLD presence.

Results Among 1672 stroke patients, 676 patients met our inclusion criteria, and 267 patients (39.5\%) had NAFLD. Compared to patients without NAFLD, patients with NAFLD were young; had high anthropometric values; high blood pressure; low aspartate aminotransferase/alanine aminotransferase ratio (AST/ALT) ratio; high levels of liver enzymes, serum albumin, HbA1c, and serum lipids; low-density lipoprotein; high serum level of some fatty acids; and high fatty acid\% of palmitic acid (PA) and dihomo-gamma-linolenic acid (DGLA). After excluding variables with multicollinearity, independent NAFLD-presence factors were high body mass index (BMI), low AST/ALT ratio, high serum albumin level, high PA\%, and high DGLA level.

Conclusions The frequency of NAFLD was high in our patient group. Significant NAFLD-presence factors were high BMI, low AST/ALT ratio, high serum albumin level, high PA\%, and high DGLA level. A further study is warranted to determine the effects of the NAFLD-presence factors on stroke onset or prevention.
\end{abstract}

Keywords Albumin · AST/ALT ratio · Blood pressure - BMI · DGLA · HbA1c $\cdot$ Hemorrhagic stroke $\cdot$ Ischemic stroke Palmitic acid $\cdot$ Predictor

\section{Introduction}

Non-alcoholic fatty liver disease (NAFLD) is emerging as one of the most common chronic liver diseases. Metabolic syndrome is a strong predictor of NAFLD that frequently occurs with obesity, type 2 diabetes mellitus, dyslipidemia,

Takahisa Mori

morit-koc@umin.net

Kazuhiro Yoshioka

y.kazuhiro12@icloud.com

Yuhei Tanno

yxip01@icloud.com

1 Department of Stroke Treatment, Shonan Kamakura General Hospital, Okamoto 1370-1, Kamakura City, Kanagawa 247-8533, Japan and hypertension [1, 2]. High body mass index (BMI), high triglyceride level, low high-density lipoprotein cholesterol (HDL) level, and high glycated hemoglobin (HbA1c) level strongly suggest the presence of NAFLD [3]. NAFLD has been reported to be a predictor of cardiovascular disease and stroke [2, 4]. Liver enzymes, such as aspartate aminotransferase (AST), alanine aminotransferase (ALT), and gammaglutamyltransferase (GGT) have been associated with the development of cardiovascular disease and all-cause mortality [5]. AST/ALT ratio is a significant predictor of chronic heart failure with reduced left ventricular ejection fraction and cardiovascular mortality [6, 7].

NAFLD may predispose one to ischemic and hemorrhagic stroke [8, 9]. A cross-sectional study conducted from 2011 to 2012 in the general Japanese population demonstrated that the prevalence of NAFLD defined by ultrasonography 
(US) was 26.4\% [10]. Previous retrospective studies reported that the prevalence of NAFLD defined by increased AST or ALT was $7.7 \%$ or $42.5 \%$, respectively, in patients with ischemic stroke [11, 12]; however, the prevalence of NAFLD diagnosed by US in patients with acute stroke is unknown. Adults aged 40-79 years have higher risks of metabolic syndrome and cardiovascular disease than individuals of other ages [13], whereas the etiology in stroke patients aged $<40$ or $\geq 80$ years is frequently different from that in patients aged 40-79 years, e.g., autoimmune disease, atrial fibrillation, or cancer-related thrombosis $[14,15]$. Stroke should be aggressively prevented in adults aged 40-79 years with metabolic syndrome or NAFLD. Dietary triglycerides (TG), which are derived from meat, fish, and vegetable oil, influence serum TG and fatty acids (FA) levels. TG, a predictor of NAFLD [10], comprises glycerol and three FAs; thus, the quality of TG depends on FAs. Elevated serum palmitic acid (PA) and oleic acid levels are associated with an increased frequency of lacunar stroke, while elevated serum docosahexaenoic acid (DHA) and arachidonic acid (AA) levels are associated with a decreased incidence of ischemic stroke [16]. Elevated serum dihomo-gamma-linolenic acid (DGLA) level and decreased DHA weight percentage (\%) of total fatty acids are associated with the onset of acute ischemic stroke at a younger age [17]. In addition, increased DGLA level and decreased eicosapentaenoic acid (EPA) percentage are associated with the onset of intracerebral hemorrhage (ICH) in young patients [18]. Dyslipidemia is related to NAFLD, and serum FAs are associated with the age of stroke onset [1, 2, 17, 18]; therefore, serum FAs may be associated with NAFLD in patients with stroke. In addition, BMI, triglycerides, type 2 diabetes mellitus, AST/ALT ratio, GGT, and blood pressure may be associated with NAFLD in patients with stroke. If the frequency of NAFLD diagnosed by US is very high in patients with acute ischemic or hemorrhagic stroke, NAFLD may be a critical risk factor for acute stroke. Therefore, identifying the factors associated with NAFLD in patients with acute stroke may lead to stroke prevention. The purpose of this retrospective cross-sectional study was to investigate whether the frequency of NAFLD diagnosed by US was high and identify the factors associated with NAFLD in patients aged 40-79 years with acute stroke.

\section{Materials and methods}

We included patients with stroke who were admitted to our institution from May 2016 to July 2019. We excluded patients who (1) were younger than 40 years or older than 79 years; (2) had an onset-to-door time of $>24 \mathrm{~h}$; (3) did not undergo body weight measurement; (4) did not undergo abdominal ultrasonography within 5 days of onset of symptom; (5) did not undergo blood examination for serum fatty acids at admission; or (6) had alcoholic fatty liver disease.

\section{Evaluation}

We evaluated age; sex; ischemic or hemorrhagic stroke; anthropometric variables, such as body weight, body height, and BMI; variables of blood pressure such as systolic blood pressure (SBP), diastolic blood pressure (DBP), and mean blood pressure; biomarkers, such as serum glucose, HbA1c, total cholesterol, low-density lipoprotein (LDL), HDL, triglycerides, AST, ALT, AST/ALT ratio, and GGT; serum concentration of FAs such as saturated fatty acids, lauric acid, myristic acid, PA, and stearic acid; $n-9$ monounsaturated fatty acids such as oleic acid; n-6 polyunsaturated fatty acids (PUFAs), such as linoleic acid (LiA), DGLA, and arachidonic acid (AA); $n$-3 PUFAs such as alpha-linolenic acid (AlA), eicosapentaenoic acid, and docosahexaenoic acid; and FA\%. LDL concentration was calculated using the Friedewald formula: $\mathrm{LDL}=$ total cholesterol - HDL - triglycerides/5. Mean blood pressure was calculated with the following formula: mean blood pressure $=(\mathrm{SBP}-\mathrm{DBP}) / 3+\mathrm{DBP}$.

\section{Measurement of fatty acids}

The levels of FAs in $1 \mathrm{~mL}$ of serum were measured at BML, Inc. (Shibuya, Tokyo, Japan). FAs were extracted using tricosanoic acid (Nu-Chek Prep, Inc., Elysian, MN, USA) as an internal standard. Lipid extracts were hydrolyzed, extracted with chloroform, and dried under nitrogen gas. After 30\% potassium methoxide methanol solution (FUJIFILM Wako Pure Chemical Corporation, Osaka, Osaka, Japan) was added to the residual sample, it was incubated at $100{ }^{\circ} \mathrm{C}$ for $5 \mathrm{~min}$, then cooled. Samples were extracted with hexane and analyzed on a GC-2010 Plus Capillary Gas Chromatograph (SHIMADZU Corporation, Kyoto, Japan) equipped with a flame ionization detector and a BPX70 column (30 $\mathrm{m} \times 0.22 \mathrm{~mm}$ I.D., $0.25-\mu \mathrm{m}$ film thickness; SHIMADZU GLC Ltd., Tokyo, Japan). Component identification was performed by comparing retention times with those of the respective standards (Sigma-Aldrich Japan, Inc., Meguro, Tokyo, Japan; Nu-Chek Prep, Inc., Elysian, MN, USA). The serum concentrations of the FAs were determined using internal standard ratios.

\section{Diagnosis of fatty liver and criterion for diagnosing "non-alcoholic" disease}

Fatty liver was diagnosed according to the findings of abdominal conventional B-mode ultrasonography performed by trained technicians. Of four known criteria (hepatorenal 
echo contrast, liver brightness, deep attenuation, and vascular blurring), hepatorenal echo contrast and liver brightness were used to diagnose fatty liver $[19,20]$. In addition, when the daily alcohol ("ethanol") consumption was lower than $30 \mathrm{~g}$ in men and $20 \mathrm{~g}$ in women, a positive NAFLD diagnosis was made [20].

\section{Statistical analysis}

The chi-square test was used to compare the categorical variables. We expressed non-normally distributed continuous variables as medians and interquartile ranges. The Wilcoxon rank-sum test was used to compare continuous variables in unpaired groups by normal approximation. We compared all possible pairs of variables with significant differences between patients with NAFLD (NAFLD presence group) and patients without NAFLD (NAFLD absence group). A dummy variable was used to represent categorical data, such as data on sex, and Spearman's rank correlation coefficient $\left(r_{\mathrm{s}}\right)$ was calculated to measure the strength of the relationships. We defined $0 \leq\left|r_{\mathrm{s}}\right|<0.1$ as no correlation, $0.1 \leq\left|r_{\mathrm{s}}\right|<0.5$ as weak correlation, and $0.5 \leq\left|r_{\mathrm{s}}\right|$ as strong correlation. Variable candidates for multiple logistic regression analyses were those with significant differences in the Wilcoxon ranksum test between the two groups. We adopted the variable candidate with a smaller or the smallest probability $(p)$ value or a larger or the largest absolute value of the Wilcoxon rank-sum test statistic ( $|z|)$ between or among variable candidates with mutually strong correlations. Multicollinearity was defined as variance inflation factor (VIF) $\geq 3$. Using variables without multicollinearity, we conducted multiple logistic regression analyses to identify independent variables that distinguish NAFLD presence from NAFLD absence. We estimated the threshold values of independent variables for NAFLD presence using the area under the curve values derived from receiver operating characteristic curves of the logistic regression model. A $p$ value $<0.05$ was considered statistically significant. We used the JMP software (version 16.0; SAS Institute, Cary, NC, USA) for all statistical analyses. One author (TM) had full access to all the data in the study and took responsibility for its integrity and the data analysis.

\section{Results}

Among 1712 patients with stroke admitted to our institution during the study period, 676 patients met our inclusion criteria for analysis (Fig. 1). Patient characteristics are summarized in Supplemental Tables 1 and 2. NAFLD was diagnosed in 267 patients (39.5\%). Compared to patients in the NAFLD absence group, patients in the NAFLD presence group were young, had high anthropometric values, had high levels of liver enzymes, had a low AST/ALT ratio, had high levels of serum albumin and $\mathrm{HbA1c}$, had high levels of serum lipids, excluding LDL, and had high BP (Table 1). Further, patients in the NAFLD presence group had high levels of serum fatty acids, except for EPA; high fatty acid\% of lauric acid, myristic acid, PA, oleic acid, and DGLA; and low fatty acid\% of stearic acid, linoleic acid, and arachidonic acid (Tables 1 and 2). There were no differences in proportions of ischemic stroke or history of statin use between the two groups (Table 1). After evaluating variables with mutually strong correlations among 36 variables with significant differences between the 2 groups (Tables 1 and 2, Supplemental Tables 3 to 12), 17 variables were adopted as variable candidates for multiple logistic regression, and VIF $<3$ in the 17 variable candidates was confirmed (Supplemental Table 13). Multiple logistic regression analysis using the 17 variables showed that BMI, AST/ALT ratio, albumin, PA\%, and DGLA were independent
Fig. 1 Flow chart of patient selection for the analysis. AFLD alcoholic fatty liver disease, $B W$ body weight, $F A$ fatty acid, NAFLD non-alcoholic fatty liver disease; US, ultrasonography

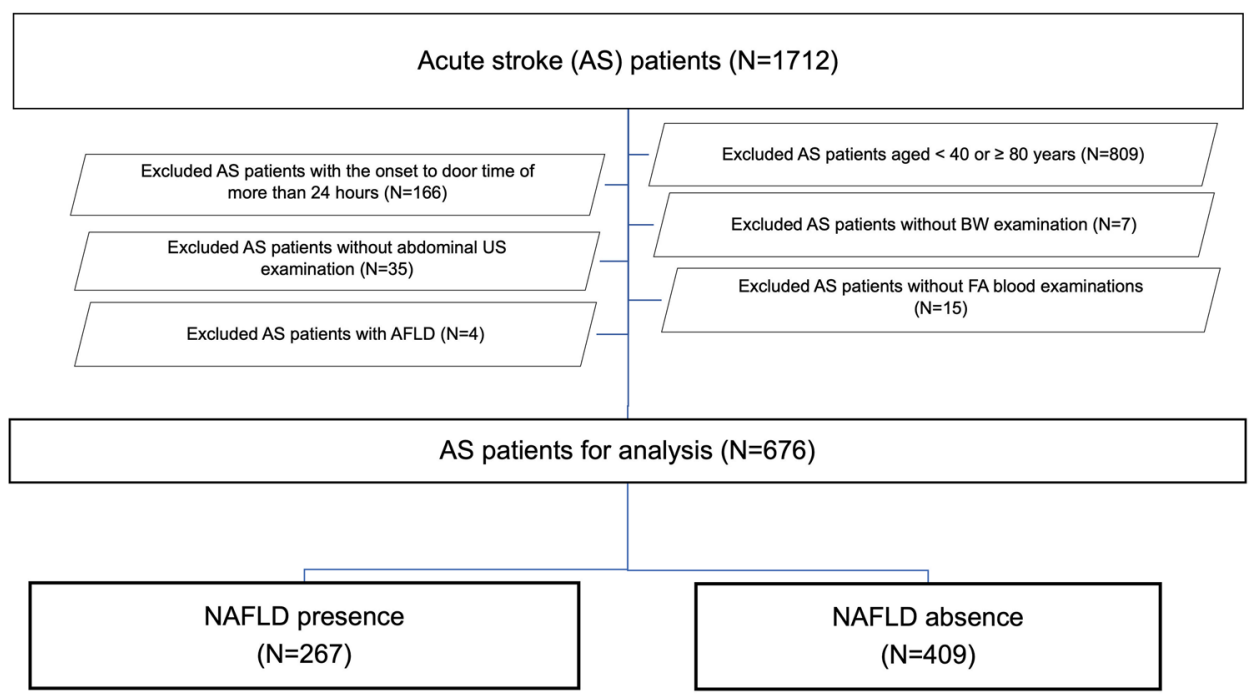


Table 1 Comparison of patients' characteristics at admission between the two groups

\begin{tabular}{|c|c|c|c|c|}
\hline & $\begin{array}{l}\text { NAFLD presence } \\
N=267\end{array}$ & $\begin{array}{l}\text { NAFLD absence } \\
N=409\end{array}$ & $|z|$ or chi & $p$ value \\
\hline Age, years & $68(60,73)$ & $71(65,76)$ & 5.17 & $<0.0001$ \\
\hline Male sex, $n(\%)$ & $184(68.9 \%)$ & $245(59.9 \%)$ & 5.71 & 0.0168 \\
\hline BMI, $\mathrm{kg} / \mathrm{m}^{2}$ & $24.8(22.3,27)$ & $21.6(19.5,24.1)$ & 10.35 & $<0.0001$ \\
\hline $\mathrm{BH}, \mathrm{cm}$ & $165(158,170)$ & $163(155,168.3)$ & 3.17 & 0.0015 \\
\hline $\mathrm{BW}, \mathrm{kg}$ & $67(58.3,77)$ & $58(50,66)$ & 9.56 & $<0.0001$ \\
\hline Ischemic stroke, $n(\%)$ & $205(76.8 \%)$ & $324(79.2 \%)$ & 0.56 & 0.4536 \\
\hline AST, U/L & $24(20,31)$ & $22(19,28)$ & 3.14 & 0.0017 \\
\hline ALT, U/L & $23(18,30)$ & $17(13,22)$ & 8.92 & $<0.0001$ \\
\hline AST/ALT ratio & $1.09(0.86,1.33)$ & $1.36(1.10,1.61)$ & 8.97 & $<0.0001$ \\
\hline GGT, U/L & $32(21,59)$ & $24(16,42.5)$ & 4.82 & $<0.0001$ \\
\hline Alb, g/L & $42(40,44)$ & $40(37.3,43)$ & 5.66 & $<0.0001$ \\
\hline Glucose, $\mathrm{mmol} / \mathrm{L}$ & $7.16(6.0,8.88)$ & $6.61(5.77,8.22)$ & 2.88 & 0.0039 \\
\hline HbA1c, \% (NGSP) & $6(5.7,6.7)$ & $5.8(5.5,6.2)$ & 4.69 & $<0.0001$ \\
\hline $\mathrm{TC}, \mathrm{mmol} / \mathrm{L}$ & $5.33(4.58,6.05)$ & $5.12(4.43,5.82)$ & 2.43 & 0.0150 \\
\hline $\mathrm{LDL}, \mathrm{mmol} / \mathrm{L}$ & $3.03(2.38,3.71)$ & $2.93(2.32,3.47)$ & 1.12 & 0.2640 \\
\hline $\mathrm{HDL}, \mathrm{mmol} / \mathrm{L}$ & $1.38(1.12,1.67)$ & $1.53(1.26,1.89)$ & 4.66 & $<0.0001$ \\
\hline $\mathrm{TG}, \mathrm{mmol} / \mathrm{L}$ & $1.52(0.93,2.64)$ & $1.05(0.78,1.57)$ & 6.62 & $<0.0001$ \\
\hline $\mathrm{SBP}, \mathrm{mmHg}$ & $170.5(151,190.3)$ & $162(141,182)$ & 3.32 & 0.0009 \\
\hline DBP, $\mathrm{mmHg}$ & $97.5(86,109.3)$ & $89(78,102)$ & 4.96 & $<0.0001$ \\
\hline $\mathrm{MBP}, \mathrm{mmHg}$ & $120.7(109.7,133.2)$ & $113(102,128.7)$ & 4.59 & $<0.0001$ \\
\hline A history of statin use, $n(\%)$ & $60(22.5 \%)$ & $96(23.5 \%)$ & 0.09 & 0.7627 \\
\hline
\end{tabular}

All values except for categorical data are represented as median (interquartile range)

Alb albumin, $A L T$ alanine aminotransferase, $A S T$ aspartate aminotransferase, $B H$ body height, $B M I$ body mass index, $B W$ body weight, chi chi-square value, $D B P$ diastolic blood pressure at admission, $G G T$ gamma-glutamyl transpeptidase, $H b A l c$ glycated hemoglobin, $H D L$ high-density lipoprotein cholesterol, $L D L$ low-density lipoprotein cholesterol, $M B P$ mean blood pressure at admission, $N A F L D$ non-alcoholic fatty liver disease, $N G S P$ National Glycohemoglobin Standardization Program, $P$ probability, $S B P$ systolic blood pressure at admission, $T C$ total cholesterol, $T G$ triglyceride, $|z|$ absolute value of the Wilcoxon ranksum test statistic
NAFLD-presence variables (Table 3). Receiver operating characteristic curves demonstrated that the threshold values of BMI, AST/ALT ratio, albumin, PA\%, and DGLA for NAFLD presence were $\geq 23.7 \mathrm{~kg} / \mathrm{m}^{2}, \leq 1.25, \geq 41 \mathrm{~g} / \mathrm{L}, \geq 24.5 \%$, and $\geq 97.1 \mu \mathrm{mol} / \mathrm{L}$, respectively (Table 4 ). These parameters (BMI, AST/ALT ratio, albumin, PA\%, and DGLA) might be NAFLD-presence risk factors at their threshold values. Of the 72 patients with no NAFLD-presence risk factors, only 5 patients $(6.9 \%)$ had NAFLD. Of the 570 patients with 1 to 4 NAFLD-presence risk factors, 232 patients had NAFLD (40.7\%; odds ratio, 9.2). Of the 34 patients with all 5 NAFLDpresence risk factors, 30 patients had NAFLD (88.2\%; odds ratio, 100.5).

\section{Discussion}

Our results demonstrate that the US-diagnosed NAFLD frequency in stroke patients aged $40-79$ years was higher than that in the general Japanese population (39.5\% vs. $26.4 \%$, respectively), and the factors associated with NAFLD presence were high BMI, low AST/ALT ratio, high albumin level, high PA\%, and high DGLA level. The NAFLD frequency was $88 \%$ in acute stroke patients with the five risk factors.

In the present study, the NAFLD frequency was very high in the middle-aged and older acute stroke patients compared to the NAFLD prevalence of $26.4 \%$ in the general Japanese population [10]. Blood pressure, glucose level, HbA1c level, total cholesterol, and triglycerides were not independent factors of NAFLD presence in acute stroke patients. Among anthropometric variables, only BMI was an independent factor of NAFLD presence in acute stroke patients. Among liver enzymes, only the AST/ALT ratio was an independent factor. The low AST/ALT ratio was also an independent factor for high BMI. Among variables of serum lipids and fatty acids, neither total cholesterol nor triglycerides, but PA\%, a saturated fatty acid, and DGLA, a member of the n-6 PUFA family, were independent NAFLD-presence factors in acute stroke patients. Eicosanoid metabolites of DGLA, such as 
Table 2 Comparison of serum fatty acids and weight percentages between the two groups

\begin{tabular}{|c|c|c|c|c|}
\hline & $\begin{array}{l}\text { NAFLD presence } \\
N=267\end{array}$ & $\begin{array}{l}\text { NAFLD absence } \\
N=409\end{array}$ & $|z|$ & $p$ value \\
\hline \multicolumn{5}{|c|}{ Saturated fatty acids } \\
\hline $\mathrm{LaA} \mu \mathrm{mol} / \mathrm{L}$ & $6.99(3.49,12.97)$ & $4.99(2.99,8.98)$ & 4.13 & $<0.0001$ \\
\hline $\mathrm{MyA} \mu \mathrm{mol} / \mathrm{L}$ & $94.4(59.9,141.1)$ & $68.3(52.1,96.8)$ & 6.18 & $<0.0001$ \\
\hline $\mathrm{PA} \mu \mathrm{mol} / \mathrm{L}$ & $2855(2321,3411)$ & $2447(2127,2812)$ & 6.63 & $<0.0001$ \\
\hline $\mathrm{StA} \mu \mathrm{mol} / \mathrm{L}$ & $728.3(601.7,835.8)$ & $656.5(550.5,766.3)$ & 4.49 & $<0.0001$ \\
\hline $\mathrm{LaA} \%$ & $0.04(0.03,0.08)$ & $0.04(0.02,0.06)$ & 2.91 & 0.0036 \\
\hline MyА \% & $0.71(0.53,0.92)$ & $0.58(0.47,0.76)$ & 5.19 & $<0.0001$ \\
\hline PA $\%$ & $23.9(22.9,25.2)$ & $23.2(22.2,24.1)$ & 6.17 & $<0.0001$ \\
\hline StA $\%$ & $6.68(6.20,7.22)$ & $6.84(6.22,7.44)$ & 2.17 & 0.0298 \\
\hline \multicolumn{5}{|l|}{ n-9 MUFA } \\
\hline $\mathrm{OlA} \mu \mathrm{mol} / \mathrm{L}$ & $2381(1846,3048)$ & $1993(1650,2509)$ & 6.60 & $<0.0001$ \\
\hline OlA \% & $22.4(20.5,24.6)$ & $20.9(19.0,23.0)$ & 6.06 & $<0.0001$ \\
\hline \multicolumn{5}{|l|}{ n-6 PUFAs } \\
\hline $\mathrm{LiA} \mu \mathrm{mol} / \mathrm{L}$ & $2757(2304,3156)$ & $2607(2220,3080)$ & 2.14 & 0.0327 \\
\hline DGLA $\mu \mathrm{mol} / \mathrm{L}$ & $111.2(86.7,136.6)$ & $86.4(68.5,110.1)$ & 7.76 & $<0.0001$ \\
\hline AA $\mu \mathrm{mol} / \mathrm{L}$ & $549.7(468.7,648.1)$ & $521.5(435.7,617.6)$ & 2.60 & 0.0092 \\
\hline $\mathrm{LiA} \%$ & $25.3(22.5,27.6)$ & $26.9(24.2,29.4)$ & 5.90 & $<0.0001$ \\
\hline DGLA $\%$ & $1.1(0.88,1.31)$ & $0.96(0.81,1.16)$ & 4.58 & $<0.0001$ \\
\hline AA $\%$ & $5.45(4.49,6.32)$ & $5.88(4.9,6.74)$ & 3.30 & 0.0010 \\
\hline \multicolumn{5}{|l|}{ n-3 PUFAs } \\
\hline $\mathrm{AlA} \mu \mathrm{mol} / \mathrm{L}$ & $75.4(54.9,105.3)$ & $65.7(49.5,93.3)$ & 3.24 & 0.0012 \\
\hline $\mathrm{EPA} \mu \mathrm{mol} / \mathrm{L}$ & $209.2(138.0,306.2)$ & $196.3(133.9,298.9)$ & 0.61 & 0.5423 \\
\hline DHA $\mu \mathrm{mol} / \mathrm{L}$ & $389.7(315.9,501.9)$ & $368.1(286.1,460.9)$ & 2.66 & 0.0078 \\
\hline $\mathrm{AlA} \%$ & $0.69(0.56,0.85)$ & $0.66(0.55,0.86)$ & 0.58 & 0.5600 \\
\hline EPA \% & $1.95(1.33,3.00)$ & $2.17(1.44,3.21)$ & 1.49 & 0.1369 \\
\hline DHA $\%$ & $4.30(3.48,5.13)$ & $4.49(3.54,5.29)$ & 1.43 & 0.1518 \\
\hline EPA/AA ratio & $0.36(0.23,0.56)$ & $0.36(0.26,0.57)$ & 0.46 & 0.6468 \\
\hline$n-6 / n-3$ ratio & $4.4(3.30,5.56)$ & $4.43(3.45,5.56)$ & 0.48 & 0.6306 \\
\hline
\end{tabular}

All values are represented as median (interquartile range)

$A A$ arachidonic acid, AlA alpha-linolenic acid, EPA eicosapentaenoic acid, DGLA dihomo-gamma-linolenic acid, DHA docosahexaenoic acid, $L a A$ lauric acid, $L i A$ linoleic acid, MyA myristic acid, OlA oleic acid, $P$ probability, $P A$ palmitic acid, StA stearic acid, NAFLD non-alcoholic fatty liver disease, $\%$ weight percentage of total fatty acids, $n-3$ PUFA n-3 polyunsaturated fatty acid, $n-6$ PUFA n-6 polyunsaturated fatty acid, $n-9$ MUFA n-9 monounsaturated fatty acid, $|z|$ absolute value of the Wilcoxon rank-sum test statistic prostaglandin E1 or thromboxane A1, have anti-inflammatory effects [21]. However, DGLA is metabolized to AA, which is metabolized to prostaglandin E2, thromboxane A2, or leukotriene $\mathrm{C} 4$ [22]; these have inflammatory effects and increase the risk of acute myocardial infarction [22]. High serum DGLA level was associated with the onset of acute ischemic stroke and ICH at a young age [17, 18] and was also associated with obesity, body fat accumulation, high ALT level, and insulin resistance in patients with type 2 diabetes [23]. High serum DGLA level was associated with not only acute stroke but also NAFLD presence. In a previous study, low fish intake was associated with high DGLA\% [24]. The dietary effect of DGLA may be more profound than that of gamma-linolenic acid, which is enzymatically elongated to DGLA [25]. Therefore, diets that contain large amounts of DGLA may increase serum DGLA levels. For example, Japanese beef cattle or crossbred (Holstein $\times$ Japanese black) beef steer contains more DGLA than imported beef or lamb [26]. However, it has not been investigated whether serum DGLA levels increase in proportion to the consumption of these proteins and whether high DGLA levels cause obesity and NAFLD.

In Japan, the National Health and Nutrition Survey reported that mean BMI slightly increased in men and women from 1995 to 2016 and the total energy intake and energy intake from proteins decreased, whereas energy intake from fats increased from 1995 to 2016 [27]. Obesity is a stubbornly obvious target for stroke prevention [28]. 
Table 3 Multiple logistic regression for NAFLD presence at the onset of stroke using receiver operating characteristic curves $(N=676)$

\begin{tabular}{llrrr}
\hline & \multicolumn{1}{l}{ Odds ratio } & \multicolumn{1}{l}{$p$ value } & AUC & BIC \\
\hline & & $<0.0001$ & 0.804 & 803 \\
BMI & $1.17(1.10-1.24)$ & $<0.0001$ & & \\
AST/ALT ratio & $0.39(0.23-0.66)$ & 0.0003 & & \\
Alb & $1.08(1.03-1.14)$ & 0.0042 & & \\
PA\% & $1.19(1.03-1.38)$ & 0.0147 & & \\
DGLA & $1.01(1.00-1.02)$ & 0.0193 & & \\
DHA & $1.00(0.99-1.00)$ & 0.1399 & & \\
HbA1c & $1.10(0.98-1.30)$ & 0.1432 & & \\
HDL & $0.76(0.45-1.23)$ & 0.3009 & & \\
GGT & $1.00(0.99-1.00)$ & 0.3987 & & \\
DBP & $1.00(0.99-1.01)$ & 0.4182 & & \\
TG & $1.09(0.88-1.37)$ & 0.4547 & & \\
MyA\% & $1.34(0.59-3.10)$ & 0.4836 & & \\
BH & $1.01(0.98-1.03)$ & 0.5109 & & \\
StA\% & $0.96(0.73-1.27)$ & 0.7937 & & \\
AA\% & $1.02(0.88-1.18)$ & 0.8224 & & \\
Age & $0.99(0.97-1.02)$ & 0.8410 & & \\
TC & $0.99(0.80-1.24)$ & 0.9482 & \\
\hline
\end{tabular}

$A A$ arachidonic acid, $A l b$ albumin, $A U C$ area under the curve, $B H$ body height, $B I C$ Bayesian information criterion, $B M I$ body mass index, $D B P$ diastolic blood pressure at admission, DGLA dihomogamma-linolenic acid, $D H A$ docosahexaenoic acid, GGT gamma-glutamyl transpeptidase, $H D L$ high-density lipoprotein cholesterol, $M y A$ myristic acid, $P$ probability, $P A$ palmitic acid, StA stearic acid, TC total cholesterol, $T G$ triglyceride, $\%$ weight percentage of total fatty acids

Therefore, a study was conducted to investigate whether the Japanese diet, characterized by high seafood and plant food composition, affects fatty acid intake and serum fatty acid\% [29]. It was found that the Japanese diet, which comprises fish, soybeans, soy products, seaweed, mushroom, konjac, and unrefined cereals, with low amounts of animal fat, meat, poultry with fat, and sweets, including deserts and snacks, was effective in increasing serum n-3 PUFA\% and decreasing serum $n$-6 PUFA\% [29]. Serum DGLA\% had a strong positive correlation with serum DGLA level (Supplemental Table 8). Therefore, the Japanese diet may decrease serum
DGLA level as a member of the $n-6$ PUFA family. The Japanese diet successfully decreased BW, BMI, and systolic and diastolic blood pressure [30]. If serum DGLA level is decreased, the incidence of NAFLD and obesity is reduced, and the incidence of an acute stroke may be prevented.

\section{Limitations}

Our study had several limitations. First, a small number of patients were included, and the study was based on a retrospective cross-sectional design without control. We compared acute stroke patients with NAFLD to those without NAFLD but did not compare them with a control population. Furthermore, a cross-sectional study cannot exclude reverse causality. Second, although ultrasonography has a sensitivity of $100 \%$ in detecting $>33 \%$ of fat, ultrasonography does not always find $\leq 33 \%$ fat and cannot distinguish steatohepatitis from simple steatosis $[19,20,31]$. Third, the patients' self-reported alcohol intake might have been biased. Fourth, because most of the patients were Japanese and there might be racial differences in the NAFLD-presence factors and the threshold values of the NAFLD-presence factors, the generalizability of the study outcomes to non-Japanese populations is uncertain. Therefore, a prospective study, including a food frequency questionnaire for detailed history of health supplement use, accurate anthropometric measurements, and biomarkers such as serum AST, ALT, albumin, fatty acid levels, and fatty acid\%, is required to determine the effects of the NAFLD-presence factors on stroke onset or prevention.

\section{Conclusions}

In acute stroke patients aged $40-79$ years, the NAFLD frequency of $39.5 \%$ at acute stroke admission was very high. The factors associated with NAFLD-presence were high BMI, low AST/ALT ratio, high albumin level, high PA\% level, and high DGLA level. A further study is warranted to determine the effects of the NAFLD-presence factors on stroke onset or prevention.
Table 4 Threshold values for NAFLD presence using receiver operating characteristic curves from logistic regression analysis $(N=676)$

\begin{tabular}{lllllll}
\hline & Sens $(\%)$ & Spec $(\%)$ & Odds ratio & $p$ value & AUC & BIC \\
\hline BMI $(\geq 23.7$ vs. $<23.7) \mathrm{kg} / \mathrm{m}^{2}$ & 65.9 & 71.4 & $1.27(1.21-1.34)$ & $<0.0001$ & 0.735 & 808 \\
AST/ALT ratio $(\leq 1.25 \mathrm{vs.}>1.25)$ & 70.4 & 63.1 & $0.18(0.11-0.28)$ & $<0.0001$ & 0.703 & 848 \\
Alb $(\geq 41$ vs. $<41) \mathrm{g} / \mathrm{L}$ & 67.0 & 52.9 & $1.11(1.07-1.16)$ & $<0.0001$ & 0.628 & 890 \\
PA\% $(\geq 24.5$ vs. $<24.5)$ & 37.6 & 84.5 & $1.39(1.25-1.55)$ & $<0.0001$ & 0.641 & 874 \\
DGLA $(\geq 97.1$ vs. $<97.1) \mu \mathrm{mol} / \mathrm{L}$ & 64.0 & 67.7 & $1.02(1.01-1.02)$ & $<0.0001$ & 0.676 & 853 \\
\hline
\end{tabular}

Alb albumin, $A L T$ alanine aminotransferase, $A S T$ aspartate aminotransferase, $A U C$ area under the curve, $B I C$ Bayesian information criterion, $B M I$ body mass index, DGLA dihomo-gamma-linolenic acid, $P$ probability, $P A$ palmitic acid, Sens sensitivity, Spec specificity, \% weight percentage of total fatty acids 
Supplementary Information The online version contains supplementary material available at https://doi.org/10.1007/s12072-021-10253-z.

Acknowledgements We would like to thank Nozomi Chiba, B.A., for her secretarial assistance at our comprehensive stroke center.

Author contributions Conceptualization: TM; methodology: TM; validation: TM; formal analysis: TM; investigation: TM, KY, YT; resources: TM, KY, YT; data curation: TM, KY, YT; writing-original draft preparation: TM; writing-review and editing: TM; visualization: TM. All authors have read and agreed to the published version of the manuscript.

Funding This research did not receive any specific grant from funding agencies in the public, commercial, or not-for-profit sectors.

Availability of data and materials The datasets generated during and/or analyzed during the current study are available from the corresponding author on reasonable request.

\section{Declarations}

Conflict of interest The authors Takahisa Mori, Kazuhiro Yoshioka and Yuhei Tanno have no relationships with any companies whose products or services may be related to the article's subject matter.

Ethics approval and consent to participate All procedures were performed in accordance with the ethical standards of the institution and the 1964 Helsinki Declaration. The Tokushukai Group Ethics Committee approved our retrospective study (TGE01402-024).

Consent to participate Written informed consent for participation was not required. Study participant enrollment was based on an opt-out model, which the ethical committee permitted.

Consent for publication Not applicable.

Open Access This article is licensed under a Creative Commons Attribution 4.0 International License, which permits use, sharing, adaptation, distribution and reproduction in any medium or format, as long as you give appropriate credit to the original author(s) and the source, provide a link to the Creative Commons licence, and indicate if changes were made. The images or other third party material in this article are included in the article's Creative Commons licence, unless indicated otherwise in a credit line to the material. If material is not included in the article's Creative Commons licence and your intended use is not permitted by statutory regulation or exceeds the permitted use, you will need to obtain permission directly from the copyright holder. To view a copy of this licence, visit http://creativecommons.org/licenses/by/4.0/.

\section{References}

1. Hamaguchi M, Kojima T, Takeda N, Nakagawa T, Taniguchi $\mathrm{H}$, Fujii K, Omatsu T, Nakajima T, Sarui H, Shimazaki M, Kato T, Okuda J, Ida K. The metabolic syndrome as a predictor of nonalcoholic fatty liver disease. Ann Intern Med 2005;143:722-728

2. Hamaguchi M, Kojima T, Takeda N, Nagata C, Takeda J, Sarui H, Kawahito Y, Yoshida N, Suetsugu A, Kato T, Okuda J, Ida $\mathrm{K}$, Yoshikawa T. Nonalcoholic fatty liver disease is a novel predictor of cardiovascular disease. World J Gastroenterol 2007;13:1579-1584
3. Sattar N, Forrest E, Preiss D. Non-alcoholic fatty liver disease. BMJ 2014;349: g4596. https://doi.org/10.1136/bmj.g4596

4. Xu J, Dai L, Zhang Y, Wang A, Li H, Wang Y, Meng X, Wu S, Wang Y. Severity of nonalcoholic fatty liver disease and risk of future ischemic stroke events. Stroke 2021;52:103-110

5. Choi KM, Han K, Park S, Chung HS, Kim NH, Yoo HJ, Seo JA, Kim SG, Kim NH, Baik SH, Park YG, Kim SM. Implication of liver enzymes on incident cardiovascular diseases and mortality: a nationwide population-based cohort study. Sci Rep 2018;8:3764. https://doi.org/10.1038/s41598-018-19700-8

6. Yokoyama M, Watanabe T, Otaki Y, Takahashi H, Arimoto T, Shishido T, Miyamoto T, Konta T, Shibata Y, Daimon M, Ueno Y, Kato T, Kayama T, Kubota I. Association of the aspartate aminotransferase to alanine aminotransferase ratio with bnp level and cardiovascular mortality in the general population: the Yamagata Study 10-year follow-up. Dis Markers 2016;2016:4857917. https://doi.org/10.1155/2016/4857917

7. Ewid M, Sherif H, Allihimy AS, Alharbi SA, Aldrewesh DA, Alkuraydis SA, Abazid R. AST/ALT ratio predicts the functional severity of chronic heart failure with reduced left ventricular ejection fraction. BMC Res Notes 2020;13:178. https://doi.org/10. 1186/s13104-020-05031-3

8. Hu J, Xu Y, He Z, Zhang H, Lian X, Zhu T, Liang C, Li J. Increased risk of cerebrovascular accident related to non-alcoholic fatty liver disease: a meta-analysis. Oncotarget 2017;9:2752-2760

9. Alkagiet S, Papagiannis A, Tziomalos K. Associations between non-alcoholic fatty liver disease and ischemic stroke. World $\mathrm{J}$ Hepatol 2018;10:474-478

10. Nishioji K, Sumida Y, Kamaguchi M, Mochizuki N, Kobayashi M, Nishimura T, Yamaguchi K, Itoh Y. Prevalence of and risk factors for non-alcoholic fatty liver disease in a non-obese Japanese population, 2011-2012. J Gastroenterol 2015;50:95-108

11. Tziomalos K, Giampatzis V, Bouziana SD, Spanou M, Papadopoulou M, Pavlidis A, Kostaki S, Bozikas A, Savopoulos C, Hatzitolios AI. Association between non-alcoholic fatty liver disease and acute ischemic stroke severity and outcome. World J Hepatol 2013;5:621-626

12. Abdeldyem SM, Goda T, Khodeir SA, Abou Saif S, Abd-Elsalam $\mathrm{S}$. Nonalcoholic fatty liver disease in patients with acute ischemic stroke is associated with more severe stroke and worse outcome. J Clin Lipidol 2017;11:915-919

13. Devers MC, Campbell S, Simmons D. Influence of age on the prevalence and components of the metabolic syndrome and the association with cardiovascular disease. BMJ Open Diabetes Res Care 2016;4: e000195. https://doi.org/10.1136/ bmjdrc-2016-000195

14. Sun LL, Tang WX, Tian M, Zhang L, Liu ZJ. Clinical manifestations and mechanisms of autoimmune disease-related multiple cerebral infarcts. Cell Transplant 2019;28:1045-1052. https://doi. org/10.1177/0963689719846838

15. Abdol Razak NB, Jones G, Bhandari M, Berndt MC, Metharom P Cancer-associated thrombosis: an overview of mechanisms, risk factors, and treatment. Cancers (Basel) 2018;10:380. https://doi. org/10.3390/cancers 10100380

16. Yaemsiri S, Sen S, Tinker LF, Robinson WR, Evans RW, Rosamond W, Wasserthiel-Smoller S, He K. Serum fatty acids and incidence of ischemic stroke among postmenopausal women. Stroke 2013;2013(44):2710-2717

17. Mori T, Yoshioka K, Tanno Y, Kasakura S. Association of serum fatty acids at admission with the age of onset of acute ischemic stroke. Nutrients 2020;12:2411. https://doi.org/10.3390/nu120 82411

18. Mori T, Yoshioka K, Tanno Y, Kasakura S. Association of serum fatty acids at admission with the age of onset of intracerebral hemorrhage. Nutrients 2020;12:2903. https://doi.org/10.3390/nu121 02903 
19. Hernaez R, Lazo M, Bonekamp S, Kamel I, Brancati FL, Guallar E, Clark JM. Diagnostic accuracy and reliability of ultrasonography for the detection of fatty liver: a meta-analysis. Hepatology 2011;54:1082-1090

20. Watanabe S, Hashimoto E, Ikejima K, Uto H, Ono M, Sumida Y, Seike M, Takei Y, Takehara T, Tokushige K, Nakajima A, Yoneda M, Saibara T, Shiota G, Sakaida I, Nakamuta M, Mizuta T, Tsubouchi H, Sugano K, Shimosegawa T. Evidence-based clinical practice guidelines for non-alcoholic fatty liver disease/nonalcoholic steatohepatitis. J Gastroenterol 2015;50:364-377

21. Fan YY, Chapkin RS. Importance of dietary gamma-linolenic acid in human health and nutrition. J Nutr 1998;128:1411-1414. https://doi.org/10.1093/jn/128.9.1411

22. Huang CC, Chang MT, Leu HB, Yin WH, Tseng WK, Wu YW, Lin TH, Yeh HI, Chang KC, Wang JH, Wu CC, Shyur LF, Chen JW. Association of arachidonic acid-derived lipid mediators with subsequent onset of acute myocardial infarction in patients with coronary artery disease. Sci Rep 2020;10:8105. https://doi.org/ 10.1038/s41598-020-65014-Z

23. Tsurutani Y, Inoue K, Sugisawa C, Saito J, Omura M, Nishikawa T. Increased serum dihomo-gamma-linolenic acid levels are associated with obesity, body fat accumulation, and insulin resistance in Japanese patients with type 2 diabetes. Intern Med 2018;57:2929-2935

24. Seah JY, Gay GM, Su J, Tai ES, Yuan JM, Koh WP, Ong CN, van Dam RM. Consumption of red meat, but not cooking oils high in polyunsaturated fat, is associated with higher arachidonic acid status in Singapore Chinese adults. Nutrients 2017;9:101. https:// doi.org/10.3390/nu9020101

25. Umeda-Sawada R, Fujiwara Y, Ushiyama I, Sagawa S, Morimitsu Y, Kawashima H, Ono Y, Kiso Y, Matsumoto A, Seyama Y. Distribution and metabolism of dihomo-gamma-linolenic acid
(DGLA, 20:3n-6) by oral supplementation in rats. Biosci Biotechnol Biochem 2006;70:2121-2130

26. Ministry of Education C, Sports, Science and Technology. Standard Tables of Food Composition in Japan (Seventh Revised Edition) - Fatty Acids. 2015. https://www.mext.go.jp/en/policy/scien ce_technology/policy/title01/detail01/sdetail01/1388555.htm

27. Saito A, Imai S, Htun NC, Okada E, Yoshita K, Yoshiike N, Takimoto $\mathrm{H}$. The trends in total energy, macronutrients and sodium intake among Japanese: findings from the 1995-2016 National Health and Nutrition Survey. Br J Nutr 2018;120:424-434

28. Kernan WN, Inzucchi SE, Sawan C, Macko RF, Furie KL. Obesity: a stubbornly obvious target for stroke prevention. Stroke 2013;44:278-286

29. Shijo Y, Maruyama C, Nakamura E, Nakano R, Shima M, Mae A, Okabe Y, Park S, Kameyama N, Hirai S. Japan diet intake changes serum phospholipid fatty acid compositions in middle-aged men: a pilot study. J Atheroscler Thromb 2019;26:3-13

30. Maruyama C, Nakano R, Shima M, Mae A, Shijo Y, Nakamura E, Okabe Y, Park S, Kameyama N, Hirai S, Nakanishi M, Uchida $\mathrm{K}$, Nishiyama $\mathrm{H}$. Effects of a Japan diet intake program on metabolic parameters in middle-aged men. $\mathrm{J}$ Atheroscler Thromb 2017;24:393-401

31. Saadeh S, Younossi ZM, Remer EM, Gramlich T, Ong JP, Hurley M, Mullen KD, Cooper JN, Sheridan MJ. The utility of radiological imaging in non-alcoholic fatty liver disease. Gastroenterology 2002; $123: 745-750$

Publisher's Note Springer Nature remains neutral with regard to jurisdictional claims in published maps and institutional affiliations. 